\title{
Short note on the stabilty of a dilatonic wall
}

\section{J. R. Morris}

Received: 11 August 2014 / Accepted: 10 September 2014 / Published online: 20 September 2014

(C) Chapman University 2014

\begin{abstract}
A nontopological soliton solution of dilaton-Maxwell theory describes a domain wall-like solution which confines magnetic flux in its core (Gibbons and Wells, Class Quant Grav 11:2499, 1994). Since the solution is not stabilized by a nontrivial topology of the vacuum manifold, it is interesting to see if the static solution is stable against small fluctuations. We consider the stability of the solution in response to small fluctuations in the scalar and magnetic fields. It is determined that the ansatz solution does indeed exhibits stability.
\end{abstract}

Keywords Dilaton-Maxwell theory $\cdot$ Domain wall $\cdot$ Stability

Mathematics Subject Classification $81 \mathrm{~T} 30 \cdot 70 \mathrm{~S} 15 \cdot 35-\mathrm{XX}$

\section{Introduction: Dilaton-Maxwell theory}

Topological solitons are stabilized by a nontrivial topology of the vacuum manifold of a scalar field, but nontopological solitons lacking a nontrivial topology of a vacuum manifold can also exist. Sometimes nontopological solitons can be stabilized by fermions (see, for example, [1-3]) or bosons (see, for example, $[4,5]$ ) that interact with or become trapped within the solitons. However, presently we focus attention on a peculiar and interesting nontopological soliton [6] that arises in dilaton-Maxwell theory, without any interactions with fermions or other scalar bosons, and attempt to determine the stability of the soliton.

We consider here the form of dilaton-Maxwell theory in a flat spacetime $\left(g_{\mu \nu}=\eta_{\mu \nu}\right)$ described by an action [6]

$S=\int \mathrm{d}^{4} x\left(\frac{1}{2} \partial_{\mu} \phi \partial^{\mu} \phi-\frac{1}{4} e^{-2 \tilde{\kappa} \phi} F_{\mu \nu} F^{\mu \nu}\right)$

J. R. Morris $(\varangle)$

Department of Physics, Indiana University Northwest, 3400 Broadway, Gary, IN 46408, USA

e-mail: jmorris@iun.edu 
where, $\tilde{\kappa}$ is an arbitrary real constant with dimension of inverse mass, so that $\tilde{\kappa} \phi$ is dimensionless, and $F_{\mu \nu}=$ $\partial_{\mu} A_{\nu}-\partial_{\nu} A_{\mu}$ is the electromagnetic field tensor. (We use a metric with negative signature $(+,-,-,-)$ ). We have

$F_{\mu \nu}=\left(\begin{array}{llll}0 & E_{x} & E_{y} & E_{z} \\ -E_{x} & 0 & -B_{z} & B_{y} \\ -E_{y} & B_{z} & 0 & -B_{x} \\ -E_{z} & -B_{y} & B_{x} & 0\end{array}\right)$

The equations of motion following from (1), along with the Bianchi identity, are

$\square \phi+\frac{1}{2} \tilde{\kappa} e^{-2 \tilde{\kappa} \phi} F_{\mu \nu} F^{\mu \nu}=0$

$\nabla_{\mu}\left(e^{-2 \tilde{\kappa} \phi} F^{\mu \nu}\right)=0, \quad \nabla_{\mu} \tilde{F}^{\mu \nu}=0$

where, $\tilde{F}_{\mu \nu}=\frac{1}{2} \epsilon_{\mu \nu \rho \sigma} F^{\rho \sigma}$ is the dual electromagnetic field tensor. The equations of (3b) can be rewritten in terms of electric and magnetic fields as

$\nabla \cdot \mathbf{D}=0, \quad \nabla \times \mathbf{H}-\dot{\mathbf{D}}=0$,

$\nabla \cdot \mathbf{B}=0, \quad \nabla \times \mathbf{E}+\dot{\mathbf{B}}=0$

with $\mathbf{D}=\epsilon \mathbf{E}$ and $\mathbf{B}=\mu \mathbf{H}$. Here the effective function $\epsilon$ and effective permeability function $\mu$ are given by

$\mu=\epsilon^{-1}=e^{2 \tilde{\kappa} \phi}$

so that the index of refraction is unity. Let us rewrite the equation of motion (3a) for the real scalar field $\phi$ in the form

$\nabla^{2} \phi-\partial_{t}^{2} \phi=-\tilde{\kappa} e^{-2 \tilde{\kappa} \phi}\left(\mathbf{B}^{2}-\mathbf{E}^{2}\right)=-\tilde{\kappa} e^{2 \tilde{\kappa} \phi}\left(\mathbf{H}^{2}-\mathbf{D}^{2}\right)$

Gibbons and Wells [6] have found a couple of interesting nontopological solitonic solutions to these equations of motion, one describing a type of cosmic string which confines magnetic field $\mathbf{B}$ and magnetic flux $\Phi_{\text {mag }}$ in its core, and another wall-like solution which also confines the magnetic field $\mathbf{B}$ and magnetic flux within its core. Here we focus upon the solitonic Gibbons-Wells wall solution to (4) and (6), which is easier to study, and consider its stability against decay.

\section{Static domain wall ansatz solution}

Gibbons and Wells discovered an interesting magnetic wall solution to (4) and (6) using an ansatz where, $\mathbf{E}=\mathbf{D}=0$ and $\mathbf{H}=(0,0, \mathcal{H})=$ const. and $\mathbf{B}=(0,0, \mathcal{B})=\mu \mathbf{H}$. Furthermore, it is assumed that the scalar field $\phi$ takes the form $\phi=\Phi(x, y)$. The equation of motion for $\Phi$ is then given by

$\left(\partial_{x}^{2}+\partial_{y}^{2}\right) \Phi=-\tilde{\kappa} \mathcal{H}^{2} e^{2 \tilde{\kappa} \Phi}$

This is recognized as the 2D Euclidean Liouville equation whose solution is given by [6-8]

$\mu(\zeta)=e^{2 \tilde{\kappa} \Phi(\zeta)}=\frac{4}{\tilde{\kappa}^{2} \mathcal{H}^{2}} \frac{\left|f^{\prime}(\zeta)\right|^{2}}{\left(1+|f(\zeta)|^{2}\right)^{2}}$ 
where, $\zeta=x+i y$ and $f(\zeta)$ is a holomorphic function of $\zeta$. For the static wall ansatz solution, $f(\zeta)$ is chosen to take the form $f(\zeta)=\exp (M \zeta)$. Then (8) yields the solution

$\mu(x)=e^{2 \tilde{\kappa} \Phi(x)}=\left(\frac{M}{\tilde{\kappa} \mathcal{H}}\right)^{2} \frac{1}{\cosh ^{2}(M x)}=\left(\frac{M}{\tilde{\kappa} \mathcal{H}}\right)^{2} \operatorname{sech}^{2}(\bar{x}), \quad \bar{x} \equiv M x$

The parameter $M$ has dimension of mass so that the coordinate $\bar{x}=M x$ is dimensionless, as is the factor $(M / \tilde{\kappa} \mathcal{H})$. Note that this ansatz solution depends only on $x$, and not on $y$.

Using (1) along with the ansatz, we write the lagrangian of this ansatz system as

$\mathcal{L}=\frac{1}{2} \partial^{\mu} \phi \partial_{\mu} \phi-\frac{1}{4} e^{-2 \tilde{\kappa} \phi} F_{\mu \nu} F^{\mu \nu}=\frac{1}{2} \partial^{\mu} \Phi \partial_{\mu} \Phi+\frac{1}{2} \mathcal{H}^{2} e^{2 \tilde{\kappa} \Phi}$

and identify the scalar potential as $V(\Phi)=-\frac{1}{2} \mathcal{H}^{2} e^{2 \tilde{\kappa} \Phi}$. We see that $\mu(x) \rightarrow 0$ and $\mathbf{B}(x) \rightarrow 0$ as $|x| \rightarrow \infty$ for the wall solution of (9). The magnetic $\mathbf{B}$ field is described by $\mathcal{B}(x)=\mu(x) \mathcal{H} \propto \operatorname{sech}^{2}(M x)$ and the magnetic flux per unit length of the wall is

$\frac{\Phi_{\text {mag }}}{L_{y}}=\frac{1}{L_{y}} \int_{-\infty}^{\infty} \int_{0}^{L_{y}} \mathcal{B}(x) \mathrm{d} x \mathrm{~d} y=\frac{2 M}{\tilde{\kappa}^{2} \mathcal{H}}$

\section{Stability}

We now consider the stability of the Gibbons-Wells magnetic wall solution of (9) in response to small fluctuations of the scalar field $\phi$ (and therefore fluctuations in B) about its ansatz value $\Phi(x)$. In particular, we want to investigate the possibility of any uncontrolled growth or decay of the ansatz solution. We write $\phi(x, y, z, t)=\Phi(x)+\delta \phi(x, y, z, t)$ and insert this into the equation of motion (6) while maintaining $\mathbf{D}=0$ and $\mathbf{B} \rightarrow \mathbf{B}^{\prime}(x, y, z, t)=\mu^{\prime}(x, y, z, t) \mathbf{H}$ with $\mathbf{H}=(0,0, \mathcal{H})=$ const and $\mu^{\prime}=\exp (\Phi(x)+\delta \phi)$. Keeping only terms linear in $\delta \phi$ and using (7) we have

$\nabla^{2} \delta \phi-\partial_{t}^{2} \delta \phi=-2(\tilde{\kappa} \mathcal{H})^{2} e^{2 \tilde{\kappa} \Phi(x)} \delta \phi$

where we have used $\left(e^{2 \tilde{\kappa} \delta \phi}-1\right)=2 \tilde{\kappa} \delta \phi$. The time dependence of $\delta \phi$ is taken to be given by $\delta \phi(x, y, z, t)=$ $\Psi(x, y, z) \sin \omega t$ and solutions of (12) with $\omega^{2}<0$ will signal an instability of the solution $\Phi(x)$, while solutions with $\omega^{2} \geq 0$ signal stability against dissipation. We therefore arrive at the equation for $\Psi(\mathbf{x})$ given by

$-\nabla^{2} \Psi-\frac{2 M^{2}}{\cosh ^{2}(\bar{x})} \Psi=\omega^{2} \Psi$

It is convenient to define the dimensionless variables $\bar{x}^{\mu}=M x^{\mu}=(\bar{t}, \bar{x}, \bar{y}, \bar{z})$ and $\bar{\omega}=\omega / M$ and rewrite (13) as

$-\bar{\nabla}^{2} \Psi(\overline{\mathbf{x}})-2 \operatorname{sech}^{2}(\bar{x}) \Psi(\overline{\mathbf{x}})=\bar{\omega}^{2} \Psi(\overline{\mathbf{x}})$

where $\bar{\nabla}^{2}$ is the Laplacian in terms of coordinates $\bar{x}, \bar{y}$, and $\bar{z}$.

We next perform a separation of variables by writing $\overline{\Psi(\mathbf{x})}=\psi(\bar{x}) f(\bar{y}) g(\bar{z})$ so that (14) leads to

$-\frac{1}{\psi} \partial_{\bar{x}}^{2} \psi-\frac{1}{f} \partial_{\bar{y}^{2}} f-\frac{1}{g} \partial_{\bar{z}^{2}} g+U(\bar{x})=\bar{\omega}^{2}$ 
with

$U(\bar{x})=-2 \operatorname{sech}^{2}(\bar{x})$

Setting $-\frac{1}{f} \partial_{\bar{y}}^{2} f=\bar{k}_{y}^{2}$ and $-\frac{1}{g} \partial_{\bar{z}}^{2} g=\bar{k}_{z}^{2}$, we have a solution of the form $\Psi(\bar{x}, \bar{y}, \bar{z}) \sim \psi(\bar{x}) \sin \left(\bar{k}_{y} \bar{y}+\delta_{y}\right) \sin \left(\bar{k}_{z} \bar{z}+\delta_{z}\right)$ where $\bar{k}_{y}^{2}+\bar{k}_{z}^{2}$ must be real valued. Now (15) reduces to

$-\partial_{\bar{x}}^{2} \psi(\bar{x})+U(\bar{x}) \psi(\bar{x})=\left[\omega^{2}-\left(\bar{k}_{y}^{2}+\bar{k}_{z}^{2}\right)\right] \psi(\bar{x})$

We now appeal to the Maxwell equations of (4) with $\mathbf{E}=\mathbf{D}=0$ with replacements $\mathbf{B} \rightarrow \mathbf{B}^{\prime}=\mu^{\prime} \mathbf{H}=$ $\mu^{\prime}(0,0, \mathcal{H})$, where

$\mu^{\prime}=e^{2 \tilde{\kappa} \phi}=e^{2 \tilde{\kappa} \Phi(x)} e^{2 \tilde{\kappa} \delta \phi}=\mu(1+2 \tilde{\kappa} \delta \phi)$

with $\mu$ given by (9). To satisfy the Maxwell equations, we require $\nabla \cdot \mathbf{B}^{\prime}=0$ and $\mathbf{B}^{\prime}=0$, which, in turn, imply that $\partial_{z} \mu^{\prime}=\partial_{z} \delta \phi=0$ and $\partial_{t} \mu^{\prime}=\partial_{t} \delta \phi=0$, requiring the conditions $\bar{k}_{z}=0$ and $\omega=0$. The result $\omega=0$ signals an absence of instability of the ansatz solution, but we must check that $\Psi$ (x) satisfies our consistency condition that $\delta \phi$ remains small for all $\mathbf{x}$.

Equation (17) has now reduced to

$-\partial_{\bar{x}}^{2} \psi(\bar{x})+U(\bar{x}) \psi(\bar{x})=-\bar{k}_{y}^{2} \psi(\bar{x}) \equiv 2 E \psi(\bar{x})$

Since $\psi(\bar{x})$ and $U(\bar{x})$ are real, we require $\bar{k}_{y}^{2}$ to be real. In order that $f(y)$ be continuous and asymptotically finite, we further require that $\bar{k}_{y}$ be real. Then we have $-\bar{k}_{y}^{2} \leq 0$. Let us set $-\bar{k}_{y}^{2}=2 E$, where $E$ is the "energy" parameter of the Schrödinger equation (19). In fact, (19) can be recognized as the Schrödinger equation with a Pöschl-Teller potential

$U(\bar{x})=-\lambda(\lambda+1) \operatorname{sech}^{2}(\bar{x})$

A comparison of (16) and (20) requires the setting $\lambda=1$. The solution to (19) for $\lambda=1$ is the bound state with "energy" $E=-1 / 2$ i.e., $\bar{k}_{y}= \pm 1$, and $\psi(\bar{x})=A \operatorname{sech}(\bar{x})$ with $A$ being a real constant. We therefore have a solution for $\delta \phi$ which can be kept small for all $\mathbf{x}$ and has $\omega=0$, satisfying the conditions for stability of the ansatz solution $\Phi(x)$ with respect to small fluctuations in the scalar field $\phi$ and magnetic field $\mathbf{B}$. In addition to the static solution $\delta \phi(x, y)$, there also exists a class of generalized magnetic wall solutions which can support arbitrarily large, nondissipating traveling waves [9] having the form $\phi(x) \rightarrow \phi(X)$, where $X=x-f(t \pm z)$ with $\square f=0$ and $\partial_{\mu} f \partial^{\mu} f=0$. (These types of "wiggly" solutions are also supported by topological domain walls and cosmic strings [10]).

In addition, we expect the wall to be stable against bending and spontaneous bubble formation. Our reasoning is that the lagrangian for the field $\phi$ is $\mathcal{L}=\frac{1}{2} \partial^{\mu} \phi \partial_{\mu} \phi-V(\phi)$, where, $V(\phi)=-\frac{1}{2} \mathcal{H}^{2} e^{2 \tilde{\kappa} \phi}$ [see (10)] and the scalar field stress-energy is $T_{\mu \nu}=\partial_{\mu} \phi \partial_{\nu} \phi-\eta_{\mu \nu}\left[\frac{1}{2} \partial^{\alpha} \phi \partial_{\alpha} \phi-V\right]$. This gives an energy density for a static wall of $T_{00}=\frac{1}{2}|\nabla \phi|^{2}+V=\frac{1}{2}|\nabla \phi|^{2}-\frac{1}{2} \mathcal{H}^{2} e^{2 \tilde{\kappa} \phi}$. Both $\phi$ and $V$ are symmetric functions of $x$, so that a slice of wall of thickness $|\delta x|$ at a distance $|x|$ from the center contributes to an amount of surface tension (energy per unit area) of magnitude $|\delta \mu|=\left|\int_{x}^{x+\delta x} T_{00} \mathrm{~d} x\right|$, which should be the same for two slices at positions $\pm|x|$. Since the surface tension is the same on both sides, i.e., there is no biasing in the energy density, we expect stability against bending, although the wall can support traveling waves [9]. 
From the ansatz solution $\Phi(x)$ given by (9), we compute the scalar field and magnetic energy densities to be

$$
\begin{aligned}
T_{00}^{(\Phi)} & =\frac{M^{2}}{2 \tilde{\kappa}^{2}}\left(\tanh ^{2} \bar{x}-\operatorname{sech}^{2} \bar{x}\right) \\
T_{00}^{(\mathbf{B})} & =\frac{1}{2} \mathbf{H} \cdot \mathbf{B}=\frac{1}{2} \mu(x) \mathcal{H}^{2}=\frac{M^{2}}{2 \tilde{\kappa}^{2}} \operatorname{sech}^{2} \bar{x}
\end{aligned}
$$

so that the total energy density is simply

$$
T_{00}^{\text {total }}=\frac{M^{2}}{2 \tilde{\kappa}^{2}} \tanh ^{2} \bar{x}
$$

which, although is finite, and minimizes in the wall's core, gives rise to a linearly divergent surface energy

$\mu\left(\left|\bar{x}_{c}\right|\right)=\frac{1}{M} \int_{-\left|\bar{x}_{c}\right|}^{+\left|\bar{x}_{c}\right|} T_{00}^{\mathrm{total}} d \bar{x}=\frac{M}{\tilde{\kappa}^{2}}\left|\bar{x}_{c}\right|$

where $\left|\bar{x}_{c}\right|$ is a large distance cut off.

\section{Summary}

We have considered the issue of stability for the Gibbons-Wells magnetic domain wall, which is unusual in that the potential for the scalar field $V(\phi)=-\frac{1}{2} \mathcal{H}^{2} e^{2 \tilde{\kappa} \phi}$, with $\mathcal{H}=$ const., is a monotonic function of $\phi$, and a normal vacuum manifold is not identified. This is in contrast to the familiar type of scalar potential with $V \sim \lambda\left(\phi^{2}-v^{2}\right)^{2}$, where vacuum states at $\phi= \pm v$ are identified and there is a $Z_{2}$ discrete symmetry with the domain wall interpolating between the two vacuum states. In that case, domain walls are stabilized by topology, whereas the Gibbons-Wells walls are nontopological. It is therefore reasonable to ask whether these magnetic walls are stable against small fluctuations. A linear perturbation analysis leads to the result that a small fluctuation $\delta \phi$ will be static $(\omega=0)$ and localized, and therefore the ansatz solutions $\Phi(x)$ and $\mathbf{B}(x)$ for the Gibbons-Wells wall are stable against decay or uncontrolled growth. Furthermore, the symmetry of $\Phi(x)$ and $\mathbf{B}(x)$, and therefore the symmetry of the energy density $T_{00}(x)$, indicates a stability against bending, since there is no biasing in the energy density.

\section{References}

1. Stojkovic, D.: Neutrino zero modes and stability of electroweak strings. Int. J. Mod. Phys. A 16(S1C), 1034-1036 (2001) e-Print: http://arxiv.org/abs/hep-th/0103216

2. Starkman, G.D., Stojkovic, D., Vachaspati, T.: Neutrino zero modes on electroweak strings. Phys. Rev. D 63, 085011 (2001) e-Print: http://arxiv.org/abs/hep-ph/0007071

3. Macpherson, A.L., Campbell, B.A.: Biased discrete symmetry breaking and Fermi balls. Phys. Lett. B 347, 205-210 (1995) e-Print: http://arxiv.org/abs/hep-ph/9408387

4. Frieman, J.A., Gelmini, G.B., Gleiser, M., Kolb, E.W.: Solitogenesis: primordial origin of nontopological solitons. Phys. Rev. Lett. 60, 2101 (1988)

5. Morris, J.R.: Stability of a class of neutral vacuum bubbles. Phys. Rev. D 87(8), 085022 (2013) (6 pp.) e-Print: http://arxiv.org/abs/ 1304.4560 [hep-th]

6. Gibbons, G.W., Wells, C.G.: Flux confinement in dilatonic cosmic strings. Class. Quant. Grav. 11, 2499-2506 (1994) e-Print: http:// arxiv.org/abs/hep-th/9312014

7. Liouville, J.: Sur l'equation aux differences partielle $\left.d^{2} \log \lambda\right) /(\operatorname{dudv}) \pm\left(\lambda /\left(2 \mathrm{a}^{2}\right)=0\right.$. J. Math. 18, $71(1853)$

8. Crowdy, D.G.: General solutions to the 2D Liouville equation. Int. J. Eng. Sci. 35, 141-149 (1997)

9. Morris, J.R.: Generalized dilaton-Maxwell cosmic string and wall solutions. Phys. Lett. B 641, 1-5 (2006) e-Print: http://arxiv.org/ abs/hep-th/0608101

10. Vachaspati, T.: Traveling waves on domain walls and cosmic strings. Phys. Lett. B 238, $41-44$ (1990) 